\title{
Ventricular tachycardia and heart failure in a patient of mid-ventricular obstructive hypertrophic cardiomyopathy with apical aneurysm: A case report
}

\author{
XIN ZHANG and XINGBIN LIU \\ Department of Cardiology, West China Hospital, Sichuan University, Chengdu, Sichuan 610041, P.R. China
}

Received September 24, 2018; Accepted February 21, 2019

DOI: $10.3892 /$ etm.2019.7796

\begin{abstract}
Mid-ventricular obstructive hypertrophic cardiomyopathy (MVOHC) with apical aneurysm is a rare type of cardiomyopathy. It is associated with an elevated risk of ventricular arrhythmias, thromboembolism, heart failure and sudden cardiac death. The present case study reports on a patient with MVOHC and apical aneurysm who developed ventricular arrhythmias and heart failure. The patient received an implantable cardioverter defibrillator for prevention of fatal arrhythmias. Ventricular tachycardia was terminated by increased doses of amiodarone and $\beta$-blocker. Transthoracic echocardiography indicated a mid-ventricular gradient of $64 \mathrm{mmHg}$. The patient refused surgical treatment and opted for alcohol septal ablation (ASA). At the 6-month follow-up, a rebound of the gradient following ASA was observed on echocardiography. In the present study, timely recognition of MVOHC with apical aneurysm led to prompt defibrillator implantation for prophylaxis regarding further malignant arrhythmias. Surgical management should be considered in symptomatic patients with MVOHC and apical aneurysm.
\end{abstract}

\section{Introduction}

Hypertrophic cardiomyopathy (HCM) is an inherited disorder of the cardiac muscle caused by a genetic defect and affects approximately one in 500 individuals (1). Patients with HCM present with multiple morphologic variants. Obstructive hypertrophic cardiomyopathy $(\mathrm{OHC})$ is a classic pathophysiological feature of HCM, characterized by unexplained left ventricular (LV) hypertrophy in association with non-dilated ventricular chambers (1). OHC is associated with an increased risk of clinical deterioration and cardiovascular mortality (1). Obstruction can cause an increase in LV systolic pressure,

Correspondence to: Dr Xingbin Liu, Department of Cardiology, West China Hospital, Sichuan University, 37 Guo Xue Xiang, Chengdu, Sichuan 610041, P.R. China

E-mail: liuxingbin@163.com

Key words: hypertrophic cardiomyopathy, apical aneurysm, ventricular tachycardia, heart failure which leads to prolonged ventricular relaxation, elevated LV diastolic pressure, mitral regurgitation, myocardial ischemia and decreased cardiac output (2-4). The presence of an aneurysmal apex in association with mid-ventricular obstruction is a rare morphological subgroup within the heterogeneous cardiomyopathy phenotypic spectrum (5). Mid-ventricular $\mathrm{OHC}$ (MVOHC) with apical aneurysm portends a wide spectrum of adverse outcomes, including ventricular arrhythmias, thromboembolism, heart failure and sudden cardiac death (6). The present study presents a rare case of MVOHC combined with apical aneurysm and its management.

\section{Case presentation}

A 61-year-old Chinese male was admitted to his local cardiology department in June 2016 due to episodes of syncope after palpitation at rest. The patient had no family history of heart disease and his electrocardiogram indicated monomorphic ventricular tachycardia (Fig. 1). The initial troponin I level was $42.96 \mathrm{ng} / \mathrm{ml}$ and the patient was diagnosed with ST-segment elevation myocardial infarction. The abnormal electrocardiogram prompted invasive coronary angiography, which provided no evidence of obstructive coronary artery disease. The electrocardiogram that was then performed exhibited sinus rhythm, ST elevation and T wave abnormality (Fig. 2). The sinus rhythm was quickly restored but could not be maintained.

After 10 days, the patient was transferred to the Department of Cardiology of West China Hospital (Chengdu, China). The electrocardiogram revealed sinus rhythm, ST elevation $(\geq 1 \mathrm{~mm}$ ) and $\mathrm{T}$ wave abnormality in V2 through V6 (negative $\mathrm{T}$ waves). Stabilization of the patient was obtained after electric cardioversion and concomitant use of $\beta$-blockers and amiodarone. Coronary angiograms indicated no narrowing of any major epicardial coronary arteries. Transthoracic echocardiography revealed normal dimensions of the LV $(48 \mathrm{~mm})$ with mid-cavity hypertrophy (maximal wall thickness in the interventricular septum, $25 \mathrm{~mm}$ ), left atrial dilatation (39 mm), a measured ejection fraction of $62 \%$ and an aneurysmal apex (Fig. 3). A subsequent cardiac acoustic contrast demonstrated clear evidence of HCM with an hourglass shape of the LV and a $35 \times 32 \mathrm{~mm}$ apical aneurysm (Fig. 4). The patient then underwent dual chamber implantable cardioverter defibrillator (ICD) implantation for prevention of fatal ventricular 


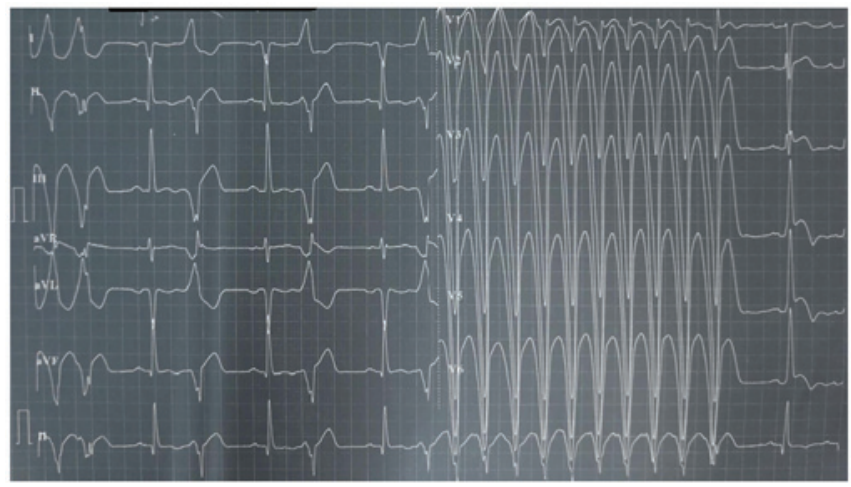

Figure 1. Twelve-lead electrocardiogram on 19th June 2016 indicating sustained monomorphic ventricular tachycardia.

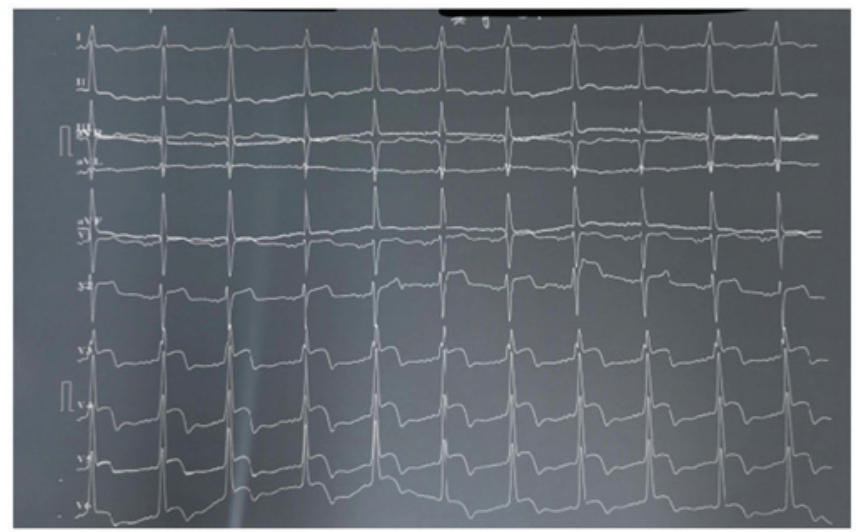

Figure 2. Twelve-lead electrocardiogram on 24th June 2016 displaying sinus rhythm, ST elevation and T wave abnormality.

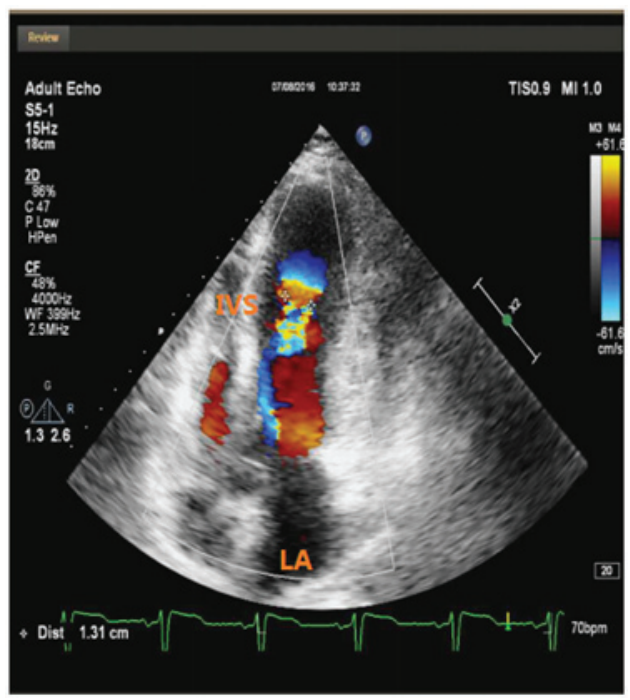

Figure 3. Transthoracic echocardiography with apical four-chamber view and color Doppler ultrasound on 8th July 2016: Severe hypertrophic cardiomyopathy with mid-cavity obstruction and apical aneurysm was indicated. LA, left atrium; IVS, intraventricular septum.

arrhythmias (Fig. 5). Subsequently, the patient remained clinically and hemodynamically stable.

One year later, the patient experienced an electrical storm with 100 episodes of sustained ventricular tachycardia

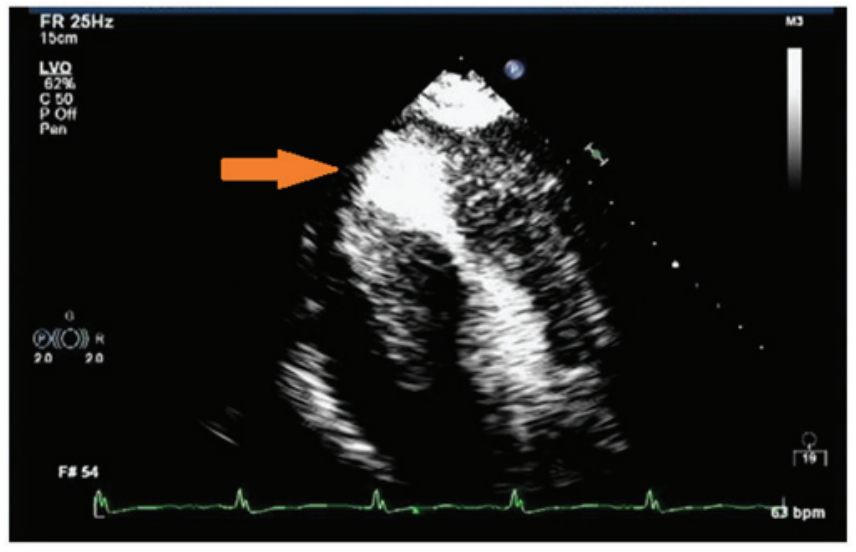

Figure 4. Contrast transthoracic echocardiographic image taken on 13th July 2016 demonstrated mid-ventricular narrowing with aneurysmal apex (arrow).

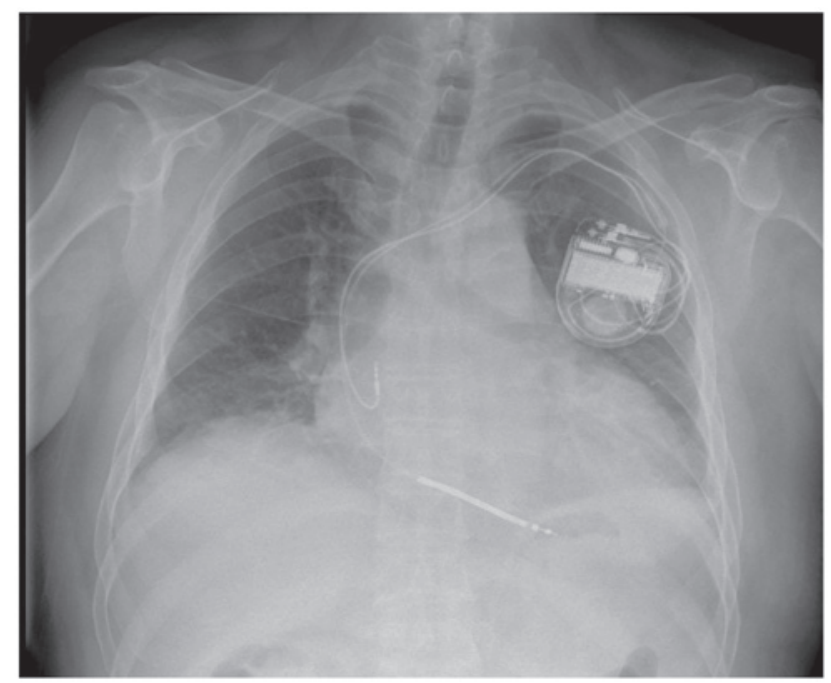

Figure 5. The X-ray performed on 20th July 2016 indicated that the patient already had a dual chamber cardioverter defibrillator implanted for prevention of fatal ventricular arrhythmias as a part of the treatment course at West China Hospital.

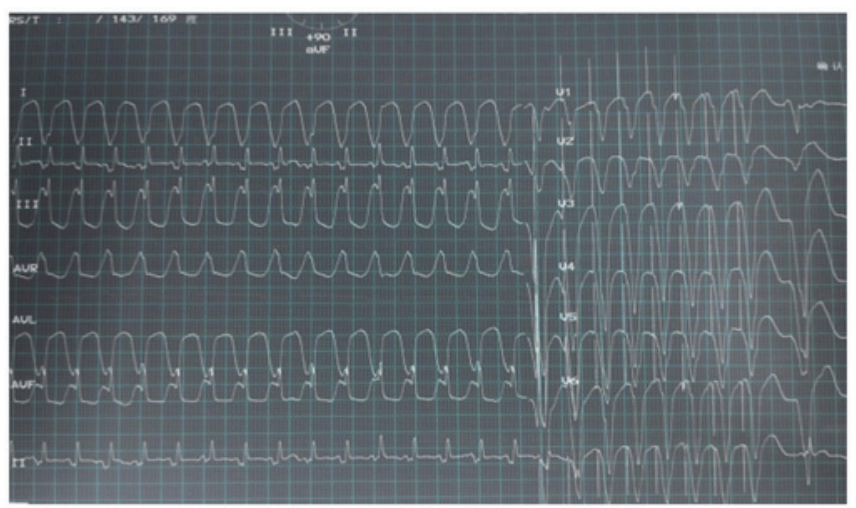

Figure 6. Twelve-lead electrocardiogram on 20th July 2017 indicating short-burst anti-tachycardia pacing followed by ventricular tachycardia.

(VT) and multiple ICD shock (Fig. 6). Recurrent sustained monomorphic VT was terminated by increased doses of amiodarone and $\beta$-blocker. Furthermore, the patient experienced 

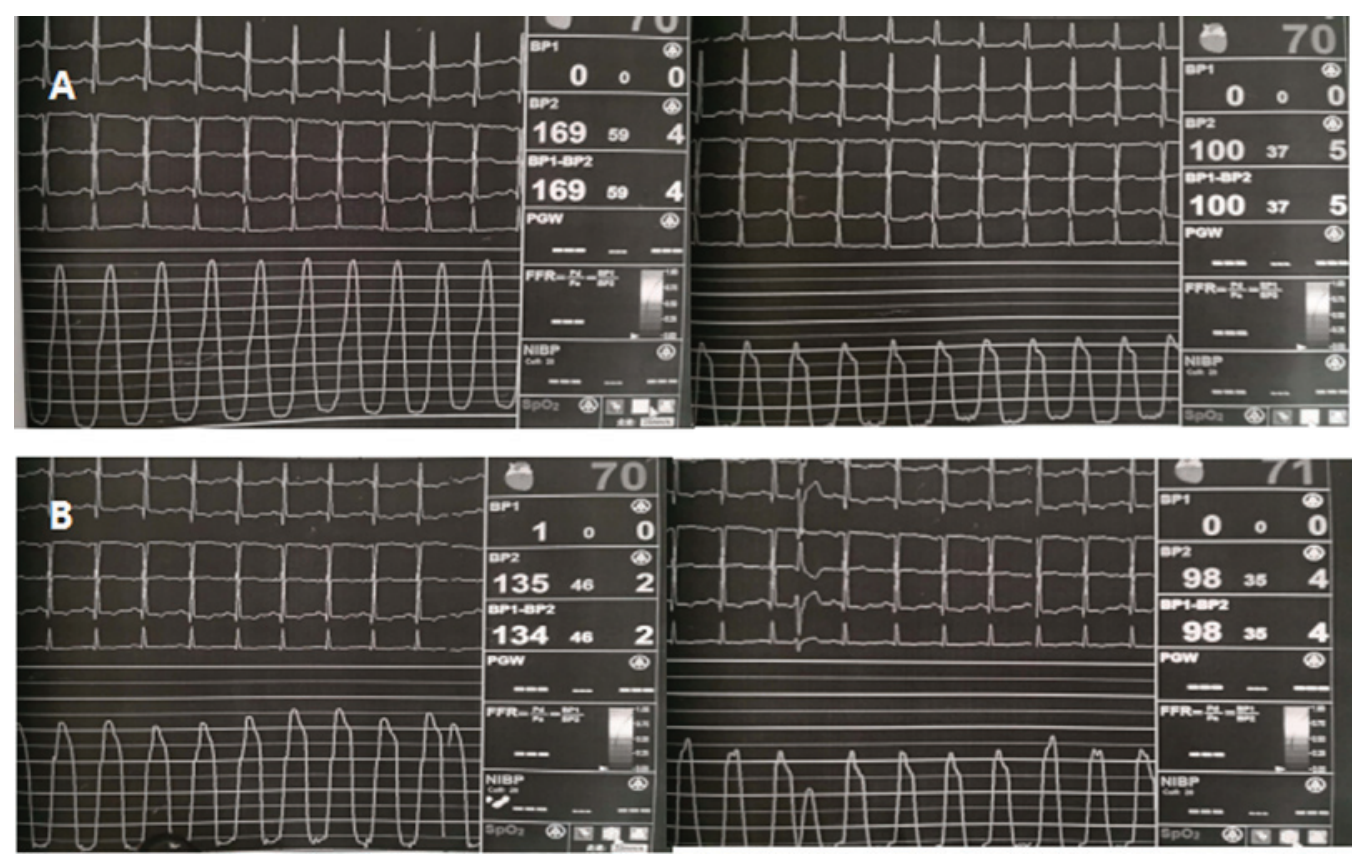

Figure 7. Percutaneous septal myocardial ablation reveling the intra-ventricular pressure gradient. (A) Pre-procedural invasive pressure recordings: (B) post-procedural invasive pressure recordings. Recordings were taken on 25th July 2017.
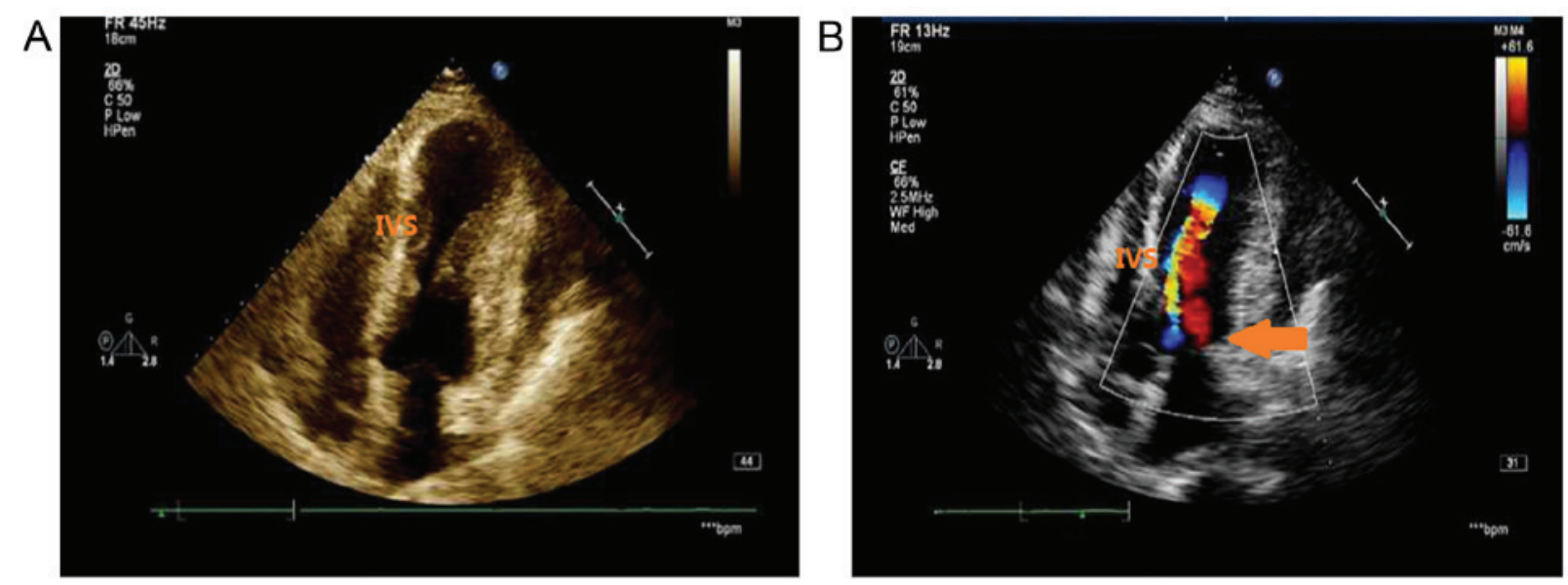

Figure 8. Echocardiographic follow-up imaging with four-chamber view on 20th January 2018. (A) The predominant thickening of the mid-ventricular and the formation of left ventricular apical aneurysm. (B) Flow turbulence caused by mid-ventricular obstruction was associated with a gradient of $80 \mathrm{mmHg}$ (arrow). IVS, intraventricular septum.

dyspnea on exertion. On transthoracic echocardiography, an apical aneurysm and a provoked mid-ventricular gradient of $64 \mathrm{mmHg}$ were noted. The patient refused surgical treatment and but provided written informed consent to undergo alcohol septal ablation. Hemodynamic assessment revealed a significant intra-ventricular pressure gradient of $69 \mathrm{mmHg}$ (LV apex, 169 mmHg; LV outflow, 100 mmHg; Fig. 7A). Repeated hemodynamic measurements after the procedure revealed a decrease of the post-extrasystolic gradient to $37 \mathrm{mmHg}$ ( $\mathrm{LV}$ apex, $135 \mathrm{mmHg}$; LV outflow, $98 \mathrm{mmHg}$ ) at the mid-ventricular level (Fig. 7B). The fourth and the fifth septal branches were dominant and echocontrast injection into target vessels resulted in the largest echocontrast view in the hypertrophic muscle segment. Therefore, the fourth and the fifth septal branches were assumed as target vessels and occlusion of these vessels resulted in a significant decrease in the intraventricular pressure gradient. The post-procedural echocardiographic examination indicated reductions in the left atrial dimension to $30 \mathrm{~mm}$, intra-ventricular septum thickness to $20 \mathrm{~mm}$ and a mid-ventricular peak systolic gradient of $19 \mathrm{mmHg}$. There were no further VT episodes during treatment with amiodarone (200 mg, once a day) and metoprolol (47.5 mg, twice per day).

At the 6-month follow-up, the patient had developed dyspnea on exertion (New York Heart Association functional class III (7). Echocardiography indicated marked intracavitary LV obstruction at the mid-ventricular level, with a peak gradient of $80 \mathrm{mmHg}$. The LV ejection fraction was $45 \%$ (Fig. 8).

\section{Discussion}

According to the 2011 American College of Cardiology Foundation (ACCF)/American Heart Association (AHA) 
guidelines, the clinical entity HCM is defined as a disease state characterized by unexplained LV hypertrophy associated with nondilated ventricular chambers in the absence of any other cardiac or systemic disease (8). Based on the electrocardiogram at the onset of the disease, indicating persistent ST-segment elevation associated with T-wave inversion in the left precordial leads, the absence of obstructive coronary artery disease and clear evidence of HCM with an apical aneurysm on Doppler echocardiography, the patient of the present study was diagnosed with MVOHC and apical aneurysm.

Development of an apical aneurysm from MVO was observed in a single-center patient cohort during the 4-year follow-up period (9). Maron et al (5) hypothesized that apical aneurysm formation and the decrease in cardiac output may be secondary to increased LV wall stress as a result of mid-cavity LV obstruction and elevated intracavitary systolic pressure. Increased wall stress imposes an increased pressure load on the apical myocardium, increasing the oxygen demand, while simultaneously reducing the coronary flow through extravascular compression of the coronary artery, leading to chronic myocardial ischemia and aneurysm formation (5). MVOHC with apical aneurysm was reported to have a poor long-term outcome, with a high risk of sudden death and potentially lethal arrhythmic events $(6,10)$. The conventional primary prevention risk markers in individual HCM patients are the following (1): i) Family history of $\geq 1$ HCM-associated sudden cardiac death; ii) $\geq 1$ episode of unexplained, recent syncope; iii) massive LV hypertrophy (thickness, $\geq 30 \mathrm{~mm}$ ); iv) unsustained VT on serial ambulatory 24-h (Holter) electrocardiograms; and v) hypotensive or attenuated blood pressure response to exercise. ICDs following cardiac arrest or sustained VT in HCM patients for secondary prevention have achieved widespread acceptance $(8,11)$. A previous study also reported that the presence of an apical aneurysm may be a novel risk factor necessitating ICD implantation (5). Considering the clinical context associated with the patient's hemodynamic instability, the patient of the present study received an ICD for prevention of fatal ventricular arrhythmias.

One year later, the patient experienced an electrical storm with 100 episodes of sustained VT and multiple ICD shock. Recurrent sustained monomorphic VT was terminated by pharmacologic therapy with increased doses of amiodarone and $\beta$-blocker. Catheter ablation is a controversial method for patients with MVOHC and apical aneurysm. VT recurrence in patients with non-ischaemic cardiomyopathy attributed to the myocardial tissue scarring of pathogenetic processes (12). A previous study reported on monomorphic VT in a case of MVOHC with apical aneurysm, and VT recurred on the next day after ablation (13). In addition, acute ablation failure is frequently associated with inability to identify the VT focus and control VT by catheter ablation (14). In the patient of the present study, ICD was implanted to prevent sudden death due to ventricular arrhythmias. Furthermore, amiodarone and $\beta$-blocker were administered to prevent arrhythmic recurrences. Therefore, the VT ablation was not performed.

According to the 2011 ACCF/AHA Guidelines for the Diagnosis and Treatment of HC, patients with marked LV outflow tract obstruction (peak instantaneous gradient at rest or with exercise, $\geq 50 \mathrm{mmHg}$ ) whose severe symptoms are refractory to maximum tolerate medical therapy and invasive relief of outflow tract obstruction should be considered (8). For
HCM with subaortic obstruction and severe drug-refractory symptoms, surgical septal myectomy should be the gold standard for septal reduction therapy in centers with comprehensive experience (15). Alcohol septal ablation (ASA) has been proposed as a less invasive alternative to surgical myectomy in patients with advanced age, those that had previously received a pacemaker or defibrillator, as well as those with significant comorbidities and a strong aversion to surgery $(16,17)$. Seggewiss and Faber (18) have reported on a case of mid-ventricular obstruction, which responded favorably to alcohol ablation. However, another previous study compared the effectiveness of ASA and transaortic extended myectomy (TEM) in HCM with MVO. The results indicated that TEM may provide a more reliable reduction in gradients compared to ASA, while serious ventricular arrhythmias were uncommon after either of the two therapies (19). Furthermore, a recent clinical study reported that surgical myectomy appeared to be protective against further malignant arrhythmias (20). Considering the high risk of surgery, the patient refused surgical treatment and underwent ASA for relief of intracavitary obstruction.

At the 6-month follow-up, rebound of the gradient following alcohol ablation was observed with an LV ejection fraction of $45 \%$ determined by echocardiography. Previous studies have reported that deterioration in LV function may due to aneurysm formation, leading to primary regional systolic impairment and cavity dilatation as a compulsory remodeling mechanism $(5,21)$. Therefore, surgical management may be a consideration in certain patients with advanced heart failure to enlarge the LV cavity.

In summary, timely recognition of MVOHC with apical aneurysm may prompt prophylactic defibrillator implantation in clinical practice for further malignant arrhythmias. Surgical management should be considered in symptomatic patients with MVOHC and apical aneurysm.

\section{Acknowledgements}

Not applicable.

\section{Funding}

No funding was received.

\section{Availability of data and materials}

The datasets used and/or analyzed during the current study are available from the corresponding author on reasonable request.

\section{Authors' contributions}

XL performed the operation and perioperative medical treatment. XL performed pre-operative examination and diagnosis. $\mathrm{XZ}$ collected the data and wrote the manuscript. All authors read and approved the final manuscript.

\section{Ethics approval and consent to participate}

Written informed consent was obtained from the participating patient. 


\section{Patient consent for publication}

Written informed consent for publication was obtained from the patient.

\section{Competing interests}

The authors declare that they have no competing interests.

\section{References}

1. Veselka J, Anavekar NS and Charron P: Hypertrophic obstructive cardiomyopathy. Lancet 389: 1253-1267, 2017.

2. Criley JM and Siegel RJ: Has 'obstruction' hindered our understanding of hypertrophic cardiomyopathy? Circulation 72 : $1148-1154,1985$

3. Maron BJ: Hypertrophic cardiomyopathy: A systematic review. JAMA 287: 1308-1320, 2002.

4. Wigle ED, Sasson Z, Henderson MA, Ruddy TD, Fulop J, Rakowski $\mathrm{H}$ and Williams WG: Hypertrophic cardiomyopathy. The importance of the site and the extent of hypertrophy. A review. Prog Cardiovasc Dis 28: 1-83, 1985.

5. Maron MS, Finley JJ, Bos JM, Hauser TH, Manning WJ, Haas TS, Lesser JR, Udelson JE, Ackerman MJ and Maron BJ: Prevalence, clinical significance, and natural history of left ventricular apical aneurysms in hypertrophic cardiomyopathy. Circulation 118 $1541-1549,2008$

6. Rowin EJ, Maron BJ, Haas TS, Garberich RF, Wang W, Link MS and Maron MS: Hypertrophic cardiomyopathy with left ventricular apical aneurysm: Implications for risk stratification and management. J Am Coll Cardiol 69: 761-773, 2017.

7. Yancy CW, Jessup M, Bozkurt B, Butler J, Casey DE Jr, Drazner MH, Fonarow GC, Geraci SA, Horwich T, Januzzi JL, et al: 2013 ACCF/AHA guideline for the management of heart failure: A report of the American College of Cardiology Foundation/American Heart Association Task Force on Practice Guidelines. J Am Coll Cardiol 62: e147-e239, 2013.

8. Gersh BJ, Maron BJ, Bonow RO, Dearani JA, Fifer MA, Link MS, Naidu SS, Nishimura RA, Ommen SR, Rakowski H, et al: 2011 ACCF/AHA guideline for the diagnosis and treatment of hypertrophic cardiomyopathy: A report of the American College of Cardiology Foundation/American Heart Association Task Force on Practice Guidelines. Circulation 124: e783-e831, 2011.

9. Minami Y, Kajimoto K, Terajima Y, Yashiro B, Okayama D, Haruki S, Nakajima T, Kawashiro N, Kawana M and Hagiwara N: Clinical implications of midventricular obstruction in patients with hypertrophic cardiomyopathy. J Am Coll Cardiol 57: 2346-2355, 2011
10. Efthimiadis GK, Pagourelias ED, Parcharidou D, Gossios T, Kamperidis V, Theofilogiannakos EK, Pappa Z, Meditskou S, Hadjimiltiades S, Pliakos C, et al: Clinical characteristics and natural history of hypertrophic cardiomyopathy with midventricular obstruction. Circ J 77: 2366-2374, 2013.

11. Authors/Task Force members, Elliott PM, Anastasakis A, Borger MA, Borggrefe M, Cecchi F, Charron P, Hagege AA, Lafont A, Limongelli G, et al: 2014 ESC Guidelines on diagnosis and management of hypertrophic cardiomyopathy: The task force for the diagnosis and management of hypertrophic cardiomyopathy of the European Society of Cardiology (ESC). Eur Heart J 35: 2733-2779, 2014.

12. Liang JJ, Santangeli P and Callans DJ: Long-term outcomes of ventricular tachycardia ablation in different types of structural heart disease. Arrhythm Electrophysiol Rev 4: 177-183, 2015.

13. Mantica M, Della Bella P and Arena V: Hypertrophic cardiomyopathy with apical aneurysm: A case of catheter and surgical therapy of sustained monomorphic ventricular tachycardia. Heart 77: 481-483, 1997

14. Tokuda M, Kojodjojo P, Tung S, Tedrow UB, Nof E, Inada K, Koplan BA, Michaud GF, John RM, Epstein LM and Stevenson WG: Acute failure of catheter ablation for ventricular tachycardia due to structural heart disease: Causes and significance. J Am Heart Assoc 2: e000072, 2013.

15. Maron BJ, Maron MS, Wigle ED and Braunwald E: The 50-year history, controversy, and clinical implications of left ventricular outflow tract obstruction in hypertrophic cardiomyopathy from idiopathic hypertrophic subaortic stenosis to hypertrophic cardiomyopathy: From idiopathic hypertrophic subaortic stenosis to hypertrophic cardiomyopathy. J Am Coll Cardiol 54: 191-200, 2009.

16. Tengiz I, Ercan E, Alioglu E and Turk UO: Percutaneous septal ablation for left mid-ventricular obstructive hypertrophic cardiomyopathy: A case report. BMC Cardiovasc Disord 6: 15, 2006.

17. Spirito P, Rossi J and Maron BJ: Alcohol septal ablation: In which patients and why? Ann Cardiothorac Surg 6: 369-375, 2017.

18. Seggewiss $\mathrm{H}$ and Faber L: Percutaneous septal ablation for hypertrophic cardiomyopathy and mid-ventricular obstruction. Eur J Echocardiogr 1: 277-280, 2000.

19. Yang YJ, Fan CM, Yuan JQ, Wang SY, Song YH, Qiao SB, You SJ, Wang ZM, Duan FJ and Li YS: Effectiveness of alcohol septal ablation versus transaortic extended myectomy in hypertrophic cardiomyopathy with midventricular obstruction. J Interv Cardiol 29: 619-627, 2016.

20. Nguyen A, Schaff HV, Nishimura RA, Dearani JA and Ommen SR: Early outcomes of repair of left ventricular apical aneurysms in patients with hypertrophic cardiomyopathy. Circulation 136: 1979-1981, 2017.

21. Opie LH, Commerford PJ, Gersh BJ and Pfeffer MA: Controversies in ventricular remodelling. Lancet 367: 356-367, 2006. 